\title{
ON SHAKEDOWN, RATCHET AND LIMIT ANALYSES OF DEFECTIVE PIPELINE
}

\author{
Haofeng Chen*, Weihang Chen, Tianbai Li, James Ure \\ Department of Mechanical Engineering, University of Strathclyde, Glasgow, G1 1XJ, UK
}

\begin{abstract}
In this study, the limit load, shakedown and ratchet limit of a defective pipeline subjected to constant internal pressure and a cyclic thermal gradient are analyzed. Ratchet limit and maximum plastic strain range are solved by employing the new Linear Matching Method (LMM) for the direct evaluation of the ratchet limit. Shakedown and ratchet limit interaction diagrams of the defective pipeline identifying the regions of shakedown, reverse plasticity, ratcheting and plastic collapse mechanism are presented and parametric studies involving different types and dimensions of part-through slot in the defective pipeline are investigated. The maximum plastic strain range over the steady cycle with different cyclic loading combinations is evaluated for a low cycle fatigue assessment. The location of the initiation of a fatigue crack for the defective pipeline with different slot type is determined. The proposed linear matching method provides a general-purpose technique for the evaluation of these key design limits and the plastic strain range for the low cycle fatigue assessment. The results for the defective pipeline shown in the paper confirm the applicability of this procedure to complex 3-D structures.
\end{abstract}

Keywords: shakedown, ratchet limit, linear matching method, defective pipeline

\section{INTRODUCTION}

Pipelines are widely used in various fields such as the petrochemical industry, energy and electric power engineering. During their operation, many local defects such as part-through slots can be produced by corrosion, mechanical damage or abrading surface cracks. These defects may jeopardize the integrity (i.e. reduce load-carrying capacity and low cycle fatigue life) of the pipelines and sometimes even lead to severe industrial accidents. The integrity assessment of defective pipelines is very important in the pipeline industry. The current testing codes and standards for the pipelines in service provide severe limitations to the allowable dimensions of part-

\footnotetext{
* Corresponding author.

Email: haofeng.chen@strath.ac.uk

Tel. +44 1415482036 Fax. +441415525105
} 
through slots. Unnecessary welding treatments of part-through slots required by the codes are not only resource-consuming processes but can also produce more severe welding defects. Therefore, some serious and systematic attempts should be made to investigate the effects of part-through slots on the load-carrying capacity and fatigue life of pipelines under cyclic loading conditions. These attempts are expected to provide some more scientific and reasonable approaches for the defect assessment and treatment. Studies of the effects of part-through slots on the load-carrying capacity of pipelines under cyclic mechanical load have been carried out [1, 2]. However, due to the lack of systematic theoretical analyses as well as enough experimental results, the effects of part-through slots on the shakedown and ratchet limit of pipelines under cyclic thermal load and a constant mechanical load are still unclear at present.

In the analysis of structures subjected to cyclic loading histories for an elastic-perfectly plastic material, the component will either shakedown or ratcheting. The elastic shakedown limit is the highest cyclic load under which a material shakes down to an elastic response after the first few load cycles. When the elastic shakedown limit is exceeded, the structure may experience either plastic shakedown or ratcheting. In many applications, it is too conservative for a structure to be within the elastic shakedown limit [3]. Plastic shakedown or alternating plasticity, under which a local low cycle fatigue failure mode occurs, may be permitted, provided that during its design life the effect of low cycle fatigue is taken into consideration. Ratcheting, which ultimately leads to incremental plastic collapse, must be avoided in any case, since it may lead to intolerable deformations. And for this reason it is desirable to calculate the ratchet limit of a structure under cyclic load condition. In addition, the evaluation of the ratchet limit is particularly useful for structures with stress raisers, such as cracks. In such structures, due to the presence of the elastic stress singularity at the crack tip the shakedown condition becomes invalid, since a finite shakedown limit does not exist anymore.

Many direct methods for modelling cyclic behaviour of the material have been developed in the past decades $[4,5,6,7,8,9]$. These direct methods use simple material models, i.e. elasticperfectly plastic, and consider a load domain that contains all possible load paths between the extremes, thus eliminating the need to know the precise load path which is normally required by the detailed step-by-step analysis [10]. Among them, the Linear Matching Method (LMM) [8,9] is recognized as one of the most powerful methods. The LMM is distinguished from the other simplified methods by ensuring that equilibrium and compatibility are satisfied at each stage $[8,9$, $11,12]$. In addition to the shakedown analysis method [11], the LMM has been extended beyond the range of most other direct methods by including the evaluation of ratchet limit and plastic strain range [8,9,12] and high temperature material behaviour [13]. The latest ratchet limit method [12] 
has been verified to be capable of evaluating the ratchet limit for defect-free components subjected to cyclic load conditions involving multi-load extremes. However, the application of this latest ratchet limit method on defective components and plastic strain range has not been undertaken.

In this paper, an extended version from the latest ratchet limit method is used in the analysis of the defective pipeline subjected to constant internal pressure and a cyclic thermal gradient. The effect of part-through slots on the load carrying capacity, shakedown and ratchet limit is presented. Parametric studies involving different types and dimensions of part through slots are carried out. ABAQUS [10] step-by-step inelastic analyses are also carried out to verify the obtained shakedown and ratchet limits by the proposed method.

\section{NUMERICAL PROCEDURES}

\subsection{Cyclic load history}

Based upon the kinematic theorem of Koiter [14] and Melan's lower bound shakedown theorem [3], the LMM procedure has proved to produce very accurate upper and lower bound shakedown limits [11] [15] [16]. The details of the LMM for limit load and shakedown limit will not be discussed here as we are primarily concerned with a new LMM for ratchet analysis. Let us consider the problem of an elastic-perfectly plastic body subjected to a general cyclic load history $F\left(x_{i}, t\right)$, which can be decomposed into cyclic component $P\left(x_{i}, t\right), \theta\left(x_{i}, t\right)$ and constant component $\lambda \bar{F}\left(x_{i}\right)$, i.e.

$$
F\left(x_{i}, t\right)=\lambda \bar{F}\left(x_{i}\right)+P\left(x_{i}, t\right)+\theta\left(x_{i}, t\right)
$$

where $\lambda$ is a load parameter, $\bar{F}\left(x_{i}\right)$ a constant load distribution, $P\left(x_{i}, t\right)$ and $\theta\left(x_{i}, t\right)$ are cyclic history of mechanical load and temperature with cycle time $\Delta t$, respectively. The variation is considered over a typical cycle $0 \leq t \leq \Delta t$ in a cyclic state. The corresponding linear elastic stress history is denoted by $\hat{\sigma}_{i j}\left(x_{k}, t\right)$ as

$$
\hat{\sigma}_{i j}\left(x_{k}, t\right)=\lambda \hat{\sigma}_{i j}^{\bar{F}}\left(x_{k}\right)+\hat{\sigma}_{i j}^{\Delta}\left(x_{k}, t\right)
$$

and

$$
\hat{\sigma}_{i j}\left(x_{k}, t\right)=\lambda \hat{\sigma}_{i j}^{\bar{F}}\left(x_{k}\right)+\hat{\sigma}_{i j}^{\Delta}\left(x_{k}, t\right)
$$

where $\hat{\sigma}_{i j} \bar{F}$ denotes the constant elastic stresses due to the constant component $\bar{F}\left(x_{i}\right)$ and $\hat{\sigma}_{i j}^{\Delta}$ denotes the varying elastic stresses due to the cyclic component $P\left(x_{i}, t\right)$ and $\theta\left(x_{i}, t\right)$.

\subsection{Asymptotic cyclic solution}


For the cyclic problem defined above, the stresses and strain rates will become asymptotic to a cyclic state where

$$
\sigma_{i j}(t)=\sigma_{i j}(t+\Delta t) \quad \dot{\varepsilon}_{i j}(t)=\dot{\varepsilon}_{i j}(t+\Delta t)
$$

The cyclic stress solution may be expressed in terms of four components, the varying elastic stress solution corresponding to the cyclic component of the load history, the associated changing residual stress field, the constant elastic stress solution due to the constant component of the load history and its associated constant residual stress. Hence, the general form of the stress solution for the cyclic problems involving changing and constant residual stress fields is given by

$$
\sigma_{i j}\left(x_{k}, t\right)=\hat{\sigma}_{i j}^{\Delta}\left(x_{k}, t\right)+\rho_{i j}^{r}\left(x_{k}, t\right)+\lambda \hat{\sigma}_{i j}^{\bar{F}}\left(x_{k}\right)+\bar{\rho}_{i j}^{\bar{F}}\left(x_{k}\right)
$$

where $\bar{\rho}_{i j} \bar{F}$ denotes a constant residual stress field in equilibrium with zero external load and corresponds to the constant component of the elastic stress history $\lambda \hat{\sigma}_{i j}^{\bar{F}}$. The $\rho_{i j}^{r}$ is the changing residual stress corresponding to the cyclic component of the elastic stress $\hat{\sigma}_{i j}^{\Delta}$ during the cycle and it satisfies the condition;

$$
\rho_{i j}^{r}\left(x_{k}, 0\right)=\rho_{i j}^{r}\left(x_{k}, \Delta t\right)=\bar{\rho}_{i j}\left(x_{k}\right)
$$

where $\bar{\rho}_{i j}\left(x_{k}\right)$ is the constant element of $\rho_{i j}^{r}$.

To evaluate the ratchet limit numerically for a component subjected to a predefined cyclic load history to withstand an extra constant load, we decouple the evaluation of the changing residual stress $\rho_{i j}^{r}(t)$ due to the cyclic part of the load and the constant residual stress $\bar{\rho}_{i j}^{\bar{F}}$ so that the varying part and constant part of the residual stress may be evaluated separately. Hence, the whole numerical procedure includes two stages; The first stage is to calculate the history of the changing residual stress field $\rho_{i j}^{r}(t)$ associated with the predefined cyclic load history and the corresponding plastic strain ranges associated with a low cycle fatigue assessment. The second stage is to locate the ratchet limit by a conventional shakedown analysis where a constant residual stress $\bar{\rho}_{i j}^{\bar{F}}$ is evaluated and the elastic stress history is augmented by the changing residual stress calculated in the first stage.

\subsection{Numerical procedure for the varying residual stress field and plastic strain range}

The Linear Matching Method procedure for the assessment of residual stress history and the associated plastic strain range due to the cyclic component of the load history is described below in 
terms of $N$ discrete time points. Following the same procedure as [13], for a strictly convex yield condition, the only instants when plastic strains can occur are at the vertices of the stress history $\hat{\sigma}_{i j}^{\Delta}\left(t_{n}\right), n=1$ to $N$, where $N$ represents the total number of time instants, $t_{1}, t_{2} \ldots . . t_{N}$, of the load extremes where plastic strain occurs and $t_{n}$ corresponds to a sequence of time points in the load history. Then the plastic strain accumulated during the cycle $\Delta \varepsilon_{i j}^{T}=\sum_{n=1}^{N} \Delta \varepsilon_{i j}^{P}\left(t_{n}\right)$ where $\Delta \varepsilon_{i j}^{P}\left(t_{n}\right)$ is the increment of plastic strain that occurs at time $t_{n}$. The entire iterative procedure includes a number of cycles, where each cycle contains $N$ iterations associated with $N$ load instances. The first iteration is to evaluate the changing residual stress $\Delta \rho_{i j}^{1}$ associated with the elastic solution $\hat{\sigma}_{i j}^{\Delta}\left(t_{1}\right)$ at the first load instance. Define $\Delta \rho_{i j}{ }_{m}$ as the evaluated changing residual stress for $n t h$ load instance at $m$ th cycle of iterations, where $n=1,2, \cdots N$ and $m=1,2 \cdots M$. At each iteration, the above changing residual stress $\Delta \rho_{i j}{ }_{m}$ for $n t h$ load instance at $m t h$ cycle of iteration is calculated. When the convergence occurs at the $m t h$ cycle of iterations, the summation of changing residual stresses at $N$ time points must approach to zero $\left(\sum_{n=1}^{N} \Delta \rho_{i j}{ }^{n}=0\right)$ due to the stable cyclic response. Hence the constant element of the residual stress for the cyclic loading history is

$$
\rho_{i j}^{r}(0)=\rho_{i j}^{r}(\Delta t)=\bar{\rho}_{i j}
$$

and determined by

$$
\bar{\rho}_{i j}=\sum_{n=1}^{N} \Delta \rho_{i j}{ }_{1}^{n}+\sum_{n=1}^{N} \Delta \rho_{i j}{ }_{2}^{n}+\cdots+\sum_{n=1}^{N} \Delta \rho_{i j}{ }_{M-1}^{n}
$$

The corresponding converged increment of plastic strain occurring at time $t_{n}$ is calculated by

$$
\Delta \varepsilon_{i j}^{p}\left(t_{n}\right)=\frac{1}{2 \bar{\mu}_{n}}\left[\bar{\sigma}_{i j}^{\Delta^{\prime}}\left(t_{n}\right)+\rho_{i j}^{\prime}\left(t_{n}\right)\right]
$$

where $\bar{\mu}_{n}$ is the iterative shear modulus and notation ( ' ) refers to the deviator component of $\bar{\sigma}_{i j}^{\Delta}$ and $\rho_{i j} \cdot \rho_{i j}\left(t_{n}\right)$ is the converged accumulated residual stress at the time instant $t_{n}$, i.e.

$$
\rho_{i j}\left(t_{n}\right)=\bar{\rho}_{i j}+\sum_{k=1}^{n} \Delta \rho_{i j_{M}}{ }^{k}
$$

The detailed iterative procedure for the evaluation of the residual stress history and associated plastic strain range has been implemented into ABAQUS through user subroutines UMAT and given in [12].

\subsection{Numerical procedure for the ratchet limit}


Once the history of the accumulated residual stress field $\rho_{i j}\left(t_{n}\right)$ at the time instance $t_{n}$ associated with the cyclic component of the load history has been calculated, the numerical technique for the ratchet limit can be accommodated within the existing method of the shakedown analysis $[11,16]$ where the linear elastic solution is augmented by the changing residual stress field $\rho_{i j}\left(t_{n}\right)$. The upper bound shakedown theorem is given by:

$$
\begin{aligned}
& \int_{0}^{\Delta t} \int_{V} \hat{\sigma}_{i j} \varepsilon_{i j}^{c} d V d t=\int_{0}^{\Delta t} \int_{V} \sigma_{i j}^{c} \varepsilon_{i j}^{c} d V d t \\
& \hat{\sigma}_{i j}=\lambda \hat{\sigma}_{i j}^{\bar{F}}+\hat{\sigma}_{i j}^{\Delta}\left(x_{k}, t\right)+\rho_{i j}^{r}\left(x_{k}, t\right)
\end{aligned}
$$

where $\varepsilon_{i j}^{c}$ is kinematically admissible strain and $\sigma_{i j}^{c}$ denotes a state of associated stress with $\varepsilon_{i j}^{c}$ at yield. For the von Mises yield condition and the associated flow rule, we have

$$
\int_{0}^{\Delta t} \int_{V} \sigma_{i j}^{c} \varepsilon_{i j}^{c} d V d t=\int_{V} \sum_{n=1}^{N} \sigma_{y} \bar{\varepsilon}\left(\Delta \varepsilon_{i j}^{n}\right) d V
$$

where $\bar{\varepsilon}\left(\Delta \varepsilon_{i j}^{n}\right)=\sqrt{\frac{2}{3} \Delta \varepsilon_{i j}^{n} \Delta \varepsilon_{i j}^{n}}$ and $\sigma_{y}$ is the yield stress of material. Thus an upper bound on the ratchet limit multiplier can be obtained by

$$
\lambda=\frac{\int_{V} \sum_{n=1}^{N} \sigma_{y} \bar{\varepsilon}\left(\Delta \varepsilon_{i j}^{n}\right) d V-\int_{V} \sum_{n=1}^{N}\left(\hat{\sigma}_{i j}^{\Delta}\left(t_{n}\right)+\rho_{i j}\left(t_{n}\right)\right) \Delta \varepsilon_{i j}^{n} d V}{\int_{V} \hat{\sigma}_{i j}^{\bar{F}}\left(\sum_{n=1}^{N} \Delta \varepsilon_{i j}^{n}\right) d V}
$$

which gives the capacity of the body subjected to a predefined cyclic load history $\hat{\sigma}_{i j}^{\Delta}\left(t_{n}\right)$ to withstand an additional constant load $\hat{\sigma}_{i j}$ before ratcheting takes place. On the basis of this formulation, the LMM produces a sequence of monotonically reducing upper bounds, which converges to the least upper bound ratchet limit for the chosen class of displacement fields. In the following sections, a defective pipeline with different types of slot is analysed in detail using the proposed method.

\section{3-D DEFECTIVE PIPELINE}

\subsection{Geometry}

The geometry [1] and the material properties of a defective pipeline subjected to constant internal pressure and a cyclic thermal gradient are shown in Fig.1 and Table 1 respectively. $R_{i}$ and $R_{0}$ are the inner radius and outer radius of the defective pipeline, respectively. The analysis is performed for different geometric parameters of a pipeline with different types of slot (Table 2). In 
all cases the inner radius and outer radius are chosen to be $R_{i}=17 \mathrm{~mm}, R_{0}=21 \mathrm{~mm}$ respectively, while the length is $L=250 \mathrm{~mm}$.

\subsection{Finite element model}

The defective pipeline is analyzed using ABAQUS type C3D20R 20 node quadratic brick elements with reduced integration scheme. The defective pipeline has two planes of symmetry. Hence, to minimize the size of the model, these symmetry boundary conditions are applied to the half section of the model. A finite element model of a defective pipeline with four different types of slot is shown in Fig. 2. The pipeline bore is under constant internal pressure. The free end of the pipeline is constrained in order to keep the plane section plane during loading. The closed-end boundary condition is achieved by applying uniform axial thrust to the end of the pipe. The uniform

axial thrust $T_{N}$ induced by the internal pressure $P$ is given by $T_{N}=P R_{i}^{2} /\left(R_{o}^{2}-R_{i}^{2}\right)$. The applied cyclic thermal loading is produced by assuming that the outside surface of the pipeline is at ambient temperature while the internal surface temperature $\theta(t)$ is fluctuating from ambient to higher values. Two thermal stress extremes are adopted for this cyclic load history:

---Firstly, a thermal stress is produced by the linear thermal gradient along the thickness. This thermal load is calculated by a steady-state thermal analysis;

---Secondly, a zero thermal stress field is selected to simulate a uniform ambient temperature for the whole defective pipeline.

The detailed temperature history at the inner surface of the defective pipeline is given in Fig.3, where $\theta(t)$ varies between $\theta_{0}$ and $\theta_{0}+\Delta \theta$. When the ambient temperature $\theta_{0}$ remains at $0^{\circ} C$, the magnitudes of the maximum thermo elastic stresses for the above thermal loading extremes can be determined by the maximum temperature difference $\Delta \theta$ between the inner surface and outer surface of the defective pipeline. Hence the cyclic thermal load and constant mechanical load can be characterized by the maximum temperature difference $\Delta \theta$ and the internal pressure $P$, respectively. The reference constant elastic mechanical stress can be calculated by the internal pressure $P=P_{0}=100 M P a$ while the reference temperature difference $\Delta \theta=\Delta \theta_{0}=100^{\circ} \mathrm{C}$ determines the reference cyclic elastic thermal stress.

\section{THE LIMIT LOAD, SHAKEDOWN AND RATCHET LIMIT INTERACTION CURVE}

The shakedown and ratchet limit interaction curve for a pipeline with small slot (shallow dimension) subjected to constant internal pressure and a cyclic thermal gradient is shown in Fig.4. The applied pressure in $\mathrm{X}$-axis is normalized with respect to the reference internal pressure $P_{0}$, 
while the thermal load in Y-axis is normalized by using the reference temperature difference $\Delta \theta=\Delta \theta_{0}=100^{\circ} \mathrm{C}$. This interaction diagram consists of shakedown limit, ratchet limit and limit load for different ratios of varying thermal load and constant mechanical load. The diagram is divided into four zones; shakedown, reverse plasticity, ratcheting and plastic collapse zone. Elastic shakedown will not occur if the load applied surpasses the reverse plasticity limit " $A B$ ". In this case the permanent strains settle into a closed cycle, a condition also known as "alternating plasticity" and associated with a low cycle fatigue mechanism. The plastic strains will increase indefinitely if the applied cyclic load level is beyond the ratchet limit "CD". This is known as "ratcheting" or “incremental plastic collapse". The point " $D$ " corresponds to the limit load for the applied mechanical load. Any applied cyclic load which exceeds the limit load line DI will cause plastic collapse.

For the verification of the ratchet limit boundary calculated by the LMM, the cyclic load points $E\left(\Delta \theta=1.5 \Delta \theta_{0}, P=0.68 P_{0}\right), F\left(\Delta \theta=3.5 \Delta \theta_{0}, P=0.45 P_{0}\right)$, and $G\left(\Delta \theta=1.5 \Delta \theta_{0}, P=0.75 P_{0}\right), H\left(\Delta \theta=3.5 \Delta \theta_{0}\right.$, $P=0.55 P_{0}$ ), which are just below and above the calculated ratchet limit boundary (Fig.4), respectively, are chosen for the step-by-step analysis by ABAQUS. The plastic strain histories for the cyclic loadings $E, G$ and $F, H$ are shown in Fig.5a and Fig.5b, respectively. From Fig.5a it is observed that the calculated maximum equivalent plastic strain for the load case $E$ exhibits shakedown as the calculated equivalent plastic strain stops changing after 3 load cycles, and the load case $G$ shows a strong ratcheting mechanism, with the maximum equivalent plastic strain increasing at every cycle. A similar result is also obtained from Fig.5b, where the calculated maximum equivalent plastic strain for the load case $F$ settles into a stable cycle after about 10 load cycles showing a reverse plasticity mechanism, and the load case $H$ shows a strong ratcheting mechanism, with the maximum equivalent plastic strain increasing at every cycle. For verifying the accuracy of the reverse plasticity limit " $A B$ ", the cyclic load points $K\left(\Delta \theta=2.3 \Delta \theta_{0}, P=0.1 P_{0}\right)$ and $L\left(\Delta \theta=2.7 \Delta \theta_{0}, P=0.1 P_{0}\right)$, which are just below and above the calculated reverse plasticity limit (Fig.4), respectively, are chosen for the step-by-step analysis by ABAQUS. Load point $K$ (Fig.5c) exhibit shakedown mechanism as the calculated equivalent plastic strain stops changing after 3 load cycles. The calculated equivalent plastic strain for the load point $L$ (Fig.5c) converges to a closed cycle after 3 load cycles showing a reverse plasticity mechanism. Thus, the results in Fig.5 obtained by ABAQUS step-by-step analysis confirm the accuracy of the predicted ratchet and shakedown limits by the LMM. Further benefits of the LMM can be found considering the computing time necessary to generate the shakedown and ratchet curves. The time that the LMM needed to generate the load points on the ratcheting boundary was less than $10 \%$ of that needed for the above cyclic load cases to complete using the ABAQUS step-by-step analyses. 


\section{RESULTS AND DISCUSSIONS}

\subsection{The effect of the part-through slot on limit load}

The loads causing plastic collapse on a defective pipeline with part-through slots and on defectfree pipeline under a constant internal pressure are shown in Table 3. From Table 3 it is observed that the calculated limit load for the defective pipeline with a small slot is identical to that for the defect-free pipeline. This reveals that the small slot does not affect the global failure mechanism of the defect-free pipeline. It can be seen from Table 3 that other types of slot cause a reduction in the limit load according to the volume of material removed. Despite removing the same volume of material, an axial slot will reduce the limit load more significantly than a circumferential slot. A thin walled pipe with closed ends subject to internal pressure will have a hoop stress which is twice the axial stress, which makes an axial slot more dangerous than a circumferential slot. The calculated limit load for the defective pipeline with a large area slot has the least value, since the material loss for this type of slot is maximum.

\subsection{The effect of the part-through slot on shakedown limit}

The shakedown and ratchet limit interaction curve for a defective pipeline with different defect types of shallow slots is shown in Fig.6. The same interaction curve with shallow and deep type slot is shown in Fig.7. In both figures the applied pressure in the X-axis is normalized with respect to the reference internal pressure while the thermal load in the Y-axis is normalized by using the reference temperature difference $\Delta \theta=\Delta \theta_{0}=100^{\circ} \mathrm{C}$. Form Fig. 6 it is observed that any part-through slot significantly reduces the reverse plasticity limit of the pipeline due to the stress concentration caused by the existence of the slot.

For a pipeline with a small slot and a pipeline with a large area slot, the reverse plasticity limits are almost identical and have a greater value than a pipeline with circumferential and axial slot. A pipeline with an axial slot has the least reverse plasticity limit due to the most significant stress concentration. In the same way as with the limit load, the axial slot has a larger impact on the hoop stress than a circumferential slot, and therefore has a larger reduction in the reverse plasticity limit. The stress concentration factor of a large area slot is less than that of an axial or circumferential slot and therefore has a larger reverse plasticity limit.

Fig.7 shows that the reverse plasticity limit decreases when the slot gets deeper. The decreasing reverse plasticity limit is due to the increasing local stress concentration in the case of a deeper slot.

\subsection{The effect of the part-through slot on ratchet limit}

From Fig. 6 it is observed that at different levels of cyclic thermal loading the ratchet limit boundary decreases sharply for a defective pipeline with axial and large area slot and it remains 
almost constant for small and circumferential slot, compared with a defect-free pipeline. This phenomenon could be explained by Fig.8, which shows the failure pattern at the ratchet limit state for a defective pipeline with a shallow slot subjected to constant internal pressure and a cyclic thermal gradient. Fig.8a and Fig.8b show that for a defective pipeline with small and circumferential slots the failure pattern appears almost in the whole body of the pipe, where the lighter colour represents the failure area. These failure pattern are a global response, which are similar to that of a defect-free pipeline. Hence the ratchet limit boundary for the pipeline with small and circumferential slots has almost the same magnitude as the defect-free pipeline. In the case of a defective pipeline with axial and large area slots (Fig.8c and Fig.8d), both failure areas appear locally around the slot, while the other parts of the pipe are unaffected. This explains why the ratchet limit boundary for the defective pipeline with axial and large area slot decreases significantly comparing to that of defect-free pipeline.

Fig. 6 also shows that for the cases of axial and large area slots, the ratchet limit ends at cyclic thermal loading points $\Delta \theta=4.1 \Delta \theta_{0}$ and $\Delta \theta=5.5 \Delta \theta_{0}$, respectively, which indicates that when the cyclic thermal loading $\Delta \theta$ beyond these cyclic thermal loading limits ( $4.1 \Delta \theta$ for axial slot and $5.5 \Delta \theta$ for large area slot), any amount of constant internal pressure will result in ratcheting.

Similar to the limit load behaviour, the results in Fig.7a show that the deeper slot has no effect on the ratchet limit boundary for the small slot type. For the circumferential slot (Fig.7b), a deeper slot reduces the ratchet limit boundary slightly. When considering the axial and large area slots (Fig.7c-7d), a deeper slot causes greater reduction in the ratchet limit boundary.

\subsection{The effect of the part-through slot on plastic strain range}

The plastic strain range concerning a fatigue crack initiation is a key factor in a low cycle fatigue assessment. The maximum plastic strain range against temperature range for different types of shallow slot subjected to cyclic thermal loading only is plotted in Fig.9a. It is observed from Fig.9a that the presence of part-through slot leads to an increase in the maximum plastic strain range. The axial slot causes a sharp increase in plastic strain range with increasing temperature compared to a defect free pipe. All other slot types cause only a small increase in comparison.

In order to investigate the effect of the constant mechanical load on the plastic strain range, three types of cyclic load histories are chosen as follows;

1) Cyclic temperature history $(\Delta \theta)$ only,

2) Cyclic temperature history and constant internal pressure $\left(\Delta \theta+0.1 P_{0}\right)$,

3) And cyclic temperature history and constant internal pressure $\left(\Delta \theta+0.2 P_{0}\right)$.

The diagrams of maximum plastic strain range versus temperature range for a defect-free pipeline and a defective pipeline with different slot types are shown in Fig.9b-Fig.9f. It can be seen 
that the plastic strain range occurs when the applied temperature range exceeds the reverse plasticity limit. The results show that the cyclic loading history with additional constant internal pressure $\left(\Delta \theta+0.1 P_{0}\right)$ causes an increase in the maximum plastic strain range. The extra increase of the constant internal pressure from $\left(0.1 P_{0}\right)$ to $\left(0.2 P_{0}\right)$ does not result in further increase in the plastic strain range. For the axial and large area slots (Figs.9e-9f), when the maximum temperature range exceeds the level $\Delta \theta=400^{\circ} \mathrm{C}$, the component will exhibit ratcheting under this cyclic temperature load and the extra constant internal pressure $\left(\Delta \theta+0.2 P_{0}\right)$ (Fig.6). Thus, for these two slot cases, the maximum plastic range is plotted for the temperature ranges up to level $\Delta \theta=400^{\circ} \mathrm{C}$.

The location of the initiation of a fatigue crack, in different types of defective pipes occurring due to fatigue of the structure under cyclic loadings, is shown in Fig.10. From Fig.10b-10d it is observed that the location of the initiation of a fatigue crack in a defective pipe with circumferential, axial or large area slots, respectively, will occur along the slot surface direction. Whereas for the defective pipe with a small slot (Fig.10a) the initiation of a fatigue crack occurs in the inner bore of the pipe.

Further investigation on this study shows that the location of the initiation of a fatigue crack for a defective pipeline is independent of the cyclic loading types considered in this paper.

\section{CONCLUSION}

In the present study, the effect of part-through slots on limit loading, shakedown limit, ratchet limit and maximum plastic strain range has been investigated using the proposed Linear Matching Method and the following observations have arisen :

1. The new Linear Matching Method has been verified by the step-by-step analysis, showing that it gives very accurate shakedown and ratchet limits for the defective pipelines with partthrough slots.

2. A defective pipeline with a small slot and circumferential shallow slot greatly reduces the thermal load at which plastic shakedown occurs but does not affect the ratchet boundary and limit load. This implies that a small slot and circumferential shallow slot of the size studied in this paper gives essentially a local stress concentration, which will affect the fatigue life of the pipeline but will not influence the gross plastic deformation or the incremental plastic collapse behaviour.

3. The presence of a part-through slot leads to an increase in the maximum plastic strain range. The maximum plastic strain ranges obtained in this study give a key information for the low cycle fatigue assessment.

4. The location of the initiation of a fatigue crack for a defective pipeline is independent of the cyclic loading types considered in this paper. 


\section{ACKNOWLEDGEMENTS}

The authors gratefully acknowledge the support of the Engineering and Physical Sciences Research Council (EP/G038880/1) of the United Kingdom, and the University of Strathclyde during the course of this work.

\section{REFERENCES}

1. Chen H. F., Liu Y. H., Cen Z. Z., Xu B. Y., 1998, "Numerical analysis of limit load and reference stress of defective pipelines under multi-loading systems," International Journal of Pressure Vessels and Piping, 75, pp.105-114

2. Chen H. F., Shu D. W., 2000, "Lower and Upper Bound Limit Analyses for Pipeline with Multi-defects of Various Configurations," International Journal of Pressure Vessels and Piping, $77, \mathrm{pp} 17-25$

3. Melan E., 1936, "Theorie Statisch Unbestimmter Systeme aus Ideal-Plastischem Bastoff. Sitzungsberichte der Akademie der Wissenschaft," Wien, Abtiia, 145, pp.195-218

4. Staat M., Heitzer M., 2001, "LISA a European Project for FEM-based Limit and Shakedown Analysis," Nuclear Engineering and Design, 206, pp.151-166

5. Vu D.K., Yan A.M., Dang Nguyen, 2004, "A primal-dual algorithm for shakedown analysis of structures,” Comput. Methods Appl. Mech. Eng, 193, pp.4663-4674

6. Seshadri R., 1995 "Inelastic Evaluation of Mechanical and Structural components Using the Generalized Local Stress Strain Method of Analysis,” Nucl. Eng. Des, 153, pp. 287-303

7. Mackenzie D., Boyle J.T., Hamilton R. , 2000, “The elastic compensation method for limit and shakedown analysis: a review," Trans IMechE, Journal of Strain Analysis for Engineering Design, 35, pp.171-188

8. Ponter A.R.S., Chen H.F., 2001, "A minimum theorem for cyclic load in excess of shakedown, with application to the evaluation of a ratchet limit," European Journal of Mechanics - A/Solids, 20, pp.539-553

9. Chen H.F., Ponter A.R.S., 2001, "A method for the evaluation of a ratchet limit and the amplitude of plastic strain for bodies subjected to cyclic loading," European Journal of Mechanics - A/Solids, 20, pp. 555-571

10. ABAQUS. 2007, User's manual. Version 6.7.

11. Chen H.F., 2010, "Lower and Upper Bound Shakedown Analysis of structures With Temperature-Dependent Yield Stress,” Journal of Pressure Vessel Technology, 132, pp.1-8 
12. Chen H.F., 2010, "A Direct Method on the Evaluation of Ratchet Limit," Journal of Pressure Vessel Technology, 132, 041202

13. Chen H.F., Ponter A.R.S., 2006, "Linear Matching Method on the evaluation of plastic and creep behaviours for bodies subjected to cyclic thermal and mechanical loading," International Journal for Numerical Methods in Engineering, 68, pp.13-32

14. Koiter W.T., 1960, “General theorems for elastic plastic solids," Progress in solid mechanics J.N.Sneddon and R.Hill, eds. North Holland, Amsterdam, 1, pp.167-221

15. Chen H.F., Ponter A.R.S., 2001, "Shakedown and limit analyses for 3-D structures using the Linear Matching Method", International Journal of Pressure Vessels and Piping, 78, pp.443451.

16. Chen H.F., Ponter A.R.S., 2001, "Shakedown and limit analyses for 3-D structures using the Linear Matching Method,” International Journal of Pressure Vessels and Piping, 78, pp.443-451 


\section{Table Captions}

Table 1 Material properties of the steel

Table 2 The pipeline shape parameters and dimensions with different defect types (shallow slot/deep slot) (mm)

Table 3 The limit loads for a pipeline with different defect types of slot under internal pressure $P_{0}$ 
Table 1. Material properties of the steel

\begin{tabular}{ccccc}
\hline Type & $\begin{array}{c}\text { Young's modulus } E \\
(\mathrm{GPa})\end{array}$ & Poisson's ratio $v$ & $\begin{array}{c}\text { Coefficient of thermal } \\
\text { expansion } \alpha\left({ }^{\circ} \mathrm{C}^{-1}\right)\end{array}$ & $\begin{array}{c}\text { Yield stress } \\
(\mathrm{MPa})\end{array}$ \\
\hline $\begin{array}{c}\text { Steel } \\
(\mathrm{S} 235 \mathrm{H})\end{array}$ & 200 & 0.3 & $1.4 \times 10^{-5}$ & 360 \\
\hline
\end{tabular}

Table 2. The pipeline shape parameters and dimensions with different defect types (shallow slot/deep slot) (mm)

\begin{tabular}{cccccc}
\hline Defect type & $\alpha$ & $\begin{array}{c}A_{1} \\
\text { Shallow/deep slot }\end{array}$ & $\begin{array}{c}A \\
\text { Shallow/deep slot }\end{array}$ & $\begin{array}{c}B \\
\text { Shallow/deep slot }\end{array}$ & $\begin{array}{c}C \\
\text { Shallow/deep slot }\end{array}$ \\
\hline Small slot & $0^{\circ}$ & $2 / 3$ & $2 / 3$ & $2 / 3$ & $2 / 3$ \\
Circumferential slot & $45^{\circ}$ & $2 / 3$ & $2 / 3$ & $2 / 3$ & $2 / 3$ \\
Axial slot & $0^{\circ}$ & $2 / 3$ & 20 & $2 / 3$ & $2 / 3$ \\
Large area slot & $45^{\circ}$ & $2 / 3$ & 20 & $2 / 3$ & $2 / 3$ \\
\hline
\end{tabular}

Table 3. The limit loads for a pipeline with different defect types of slot under internal pressure $P_{0}$

\begin{tabular}{ccc}
\hline Defect type & $\begin{array}{c}\text { Limit Load for shallow type } \\
\text { slot (MPa) }\end{array}$ & $\begin{array}{c}\text { Limit Load for deep type slot } \\
\text { (MPa) }\end{array}$ \\
\hline Defect-free & 87.8 & 87.8 \\
Small slot & 87.8 & 87.7 \\
Circumferential slot & 87.4 & 72.3 \\
Axial slot & 62.6 & 46.2 \\
Large area slot & 49.1 & 24.4 \\
\hline
\end{tabular}




\section{Figure Captions}

Fig.1 The geometry of a pipeline with part-through slot subjected to internal pressure and cyclic thermal load

Fig.2 The finite element mesh for a pipeline with part-through slot: (a) small slot; (b) circumferential slot; (c) axial slot and (d) large area slot

Fig.3 The cyclic thermal loading history for defective pipeline

Fig.4 The ratchet limit boundary for small slot case

Fig.5 ABAQUS verification using step by step analysis for: (a) the shakedown and ratchet limit and (b) reverse plasticity and ratchet limit (c) reverse plasticity limit " $A B$ "

Fig.6 Shakedown and ratchet limit interaction curve for defective pipeline with shallow type slot

Fig.7 Shakedown and ratchet limit interaction curve of part-through slot with different dimensions: (a)small slot; (b) circumferential slot; (c) axial slot and (d) large area slot

Fig.8 Failure pattern at the limit state for defective pipeline: (a) small slot; (b) circumferential slot; (c)axial slot and (d)large area slot

Fig.9 Maximum equivalent plastic strain range against temperature range for : (a) Cyclic thermal load only ( all defect pipeline); (b)Cyclic thermal and mechanical load (defect-free); (c) Cyclic thermal and mechanical load (small slot); (d) Cyclic thermal and mechanical load (circumferential slot); (e) Cyclic thermal and mechanical load (axial slot); (f) Cyclic thermal and mechanical load (large area slot)

Fig.10 The location of the initiation of a fatigue crack under cyclic thermal load and constant internal pressure (a)small slot; (b) circumferential slot; (c) axial slot and (d) large area slot 


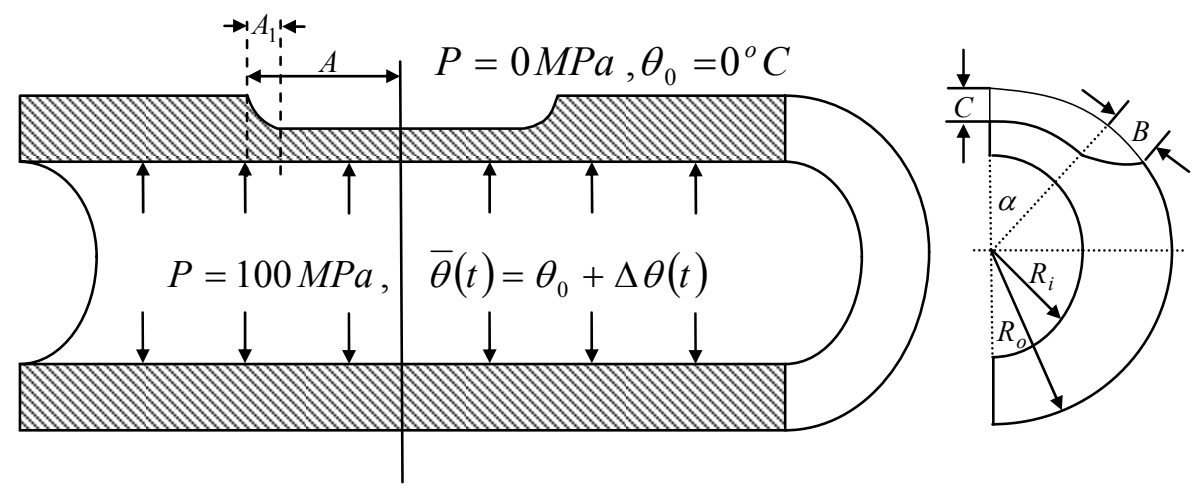

Fig. 1 The geometry of a pipeline with part-through slot subjected to internal pressure and cyclic thermal load 


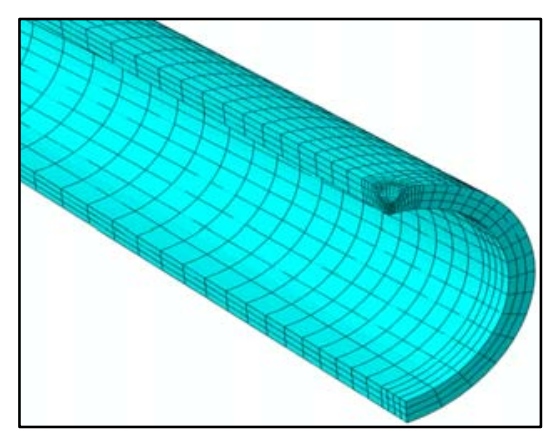

(a) Small slot

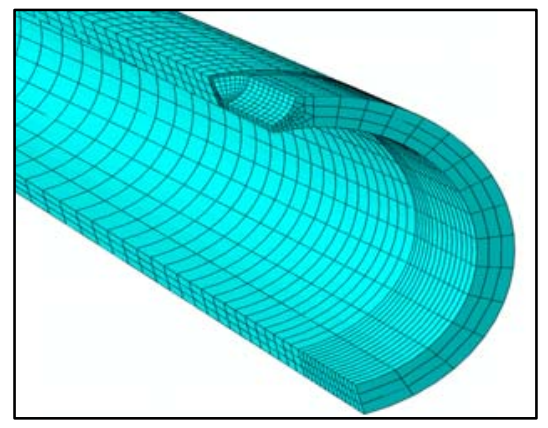

(c) Axial slot

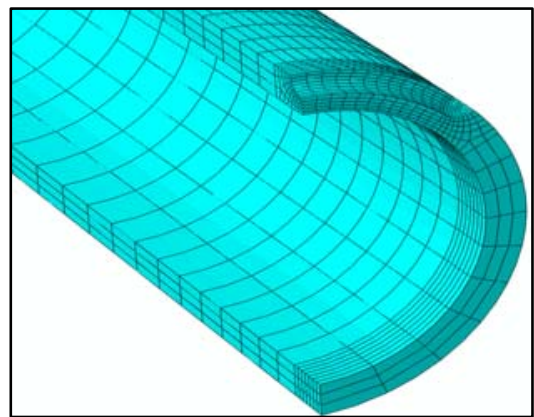

(b) Circumferential slot

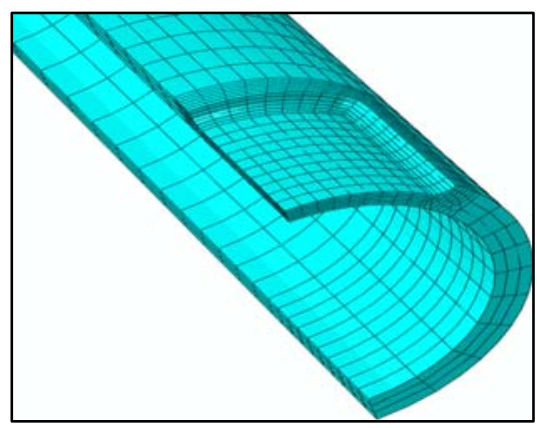

(d) Large area slot

Fig. 2 The finite element mesh for a pipeline with part-through slot: (a) small slot; (b) circumferential slot; (c) axial slot and (d) large area slot 


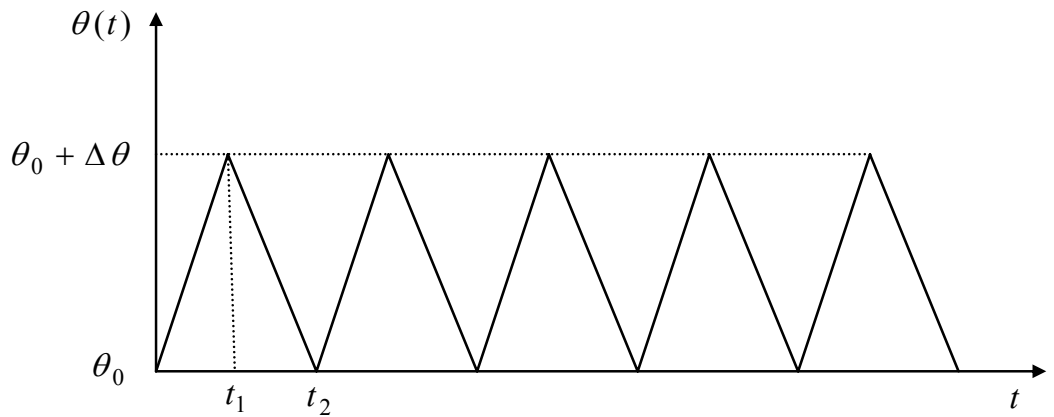

Fig. 3 The cyclic thermal loading history for defective pipeline 


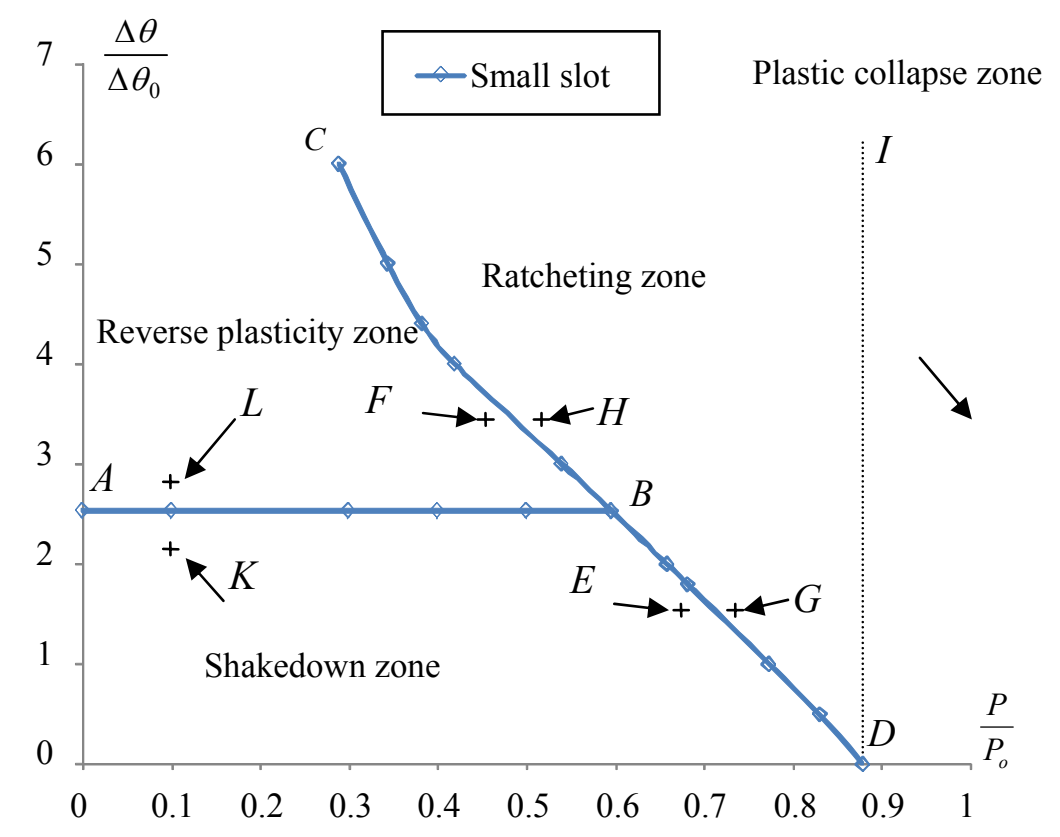

Fig. 4 The ratchet limit boundary for small slot case 


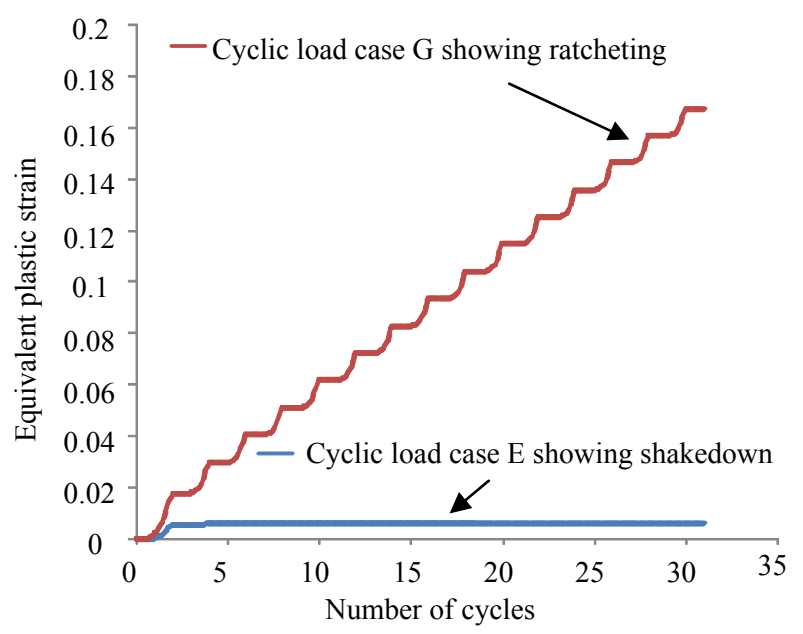

(a)

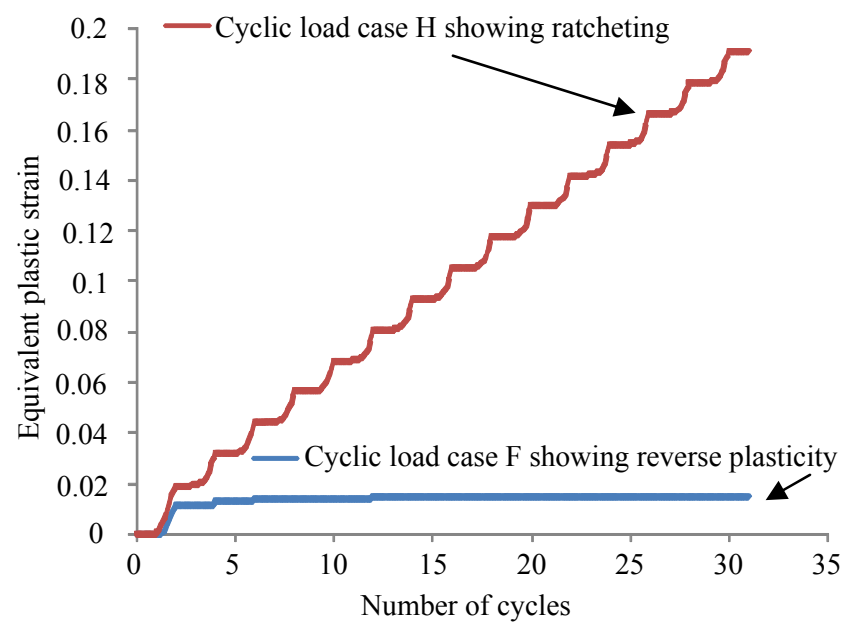

(b)

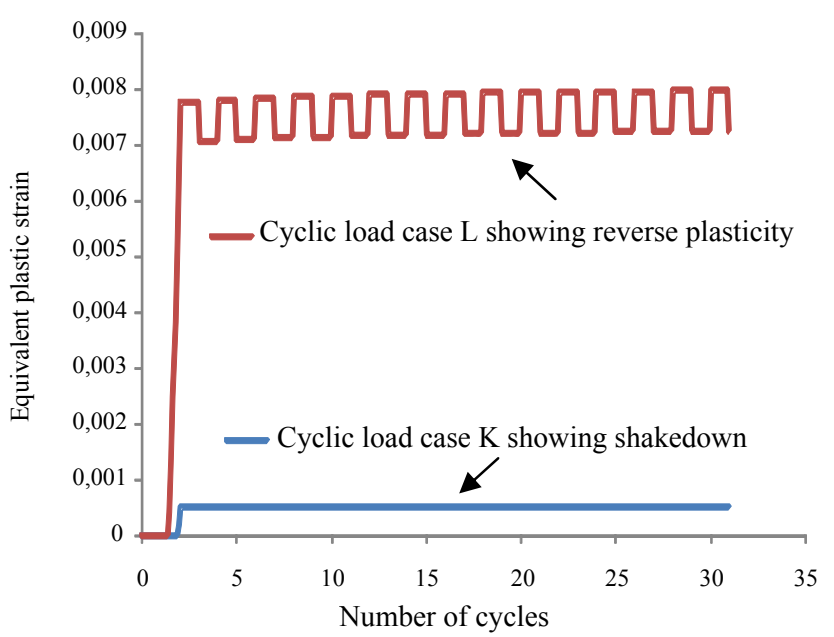

(c)

Fig. 5 ABAQUS verification using step by step analysis for: (a) the shakedown and ratchet limit and (b) reverse plasticity and ratchet limit (c) reverse plasticity limit " $A B$ " 


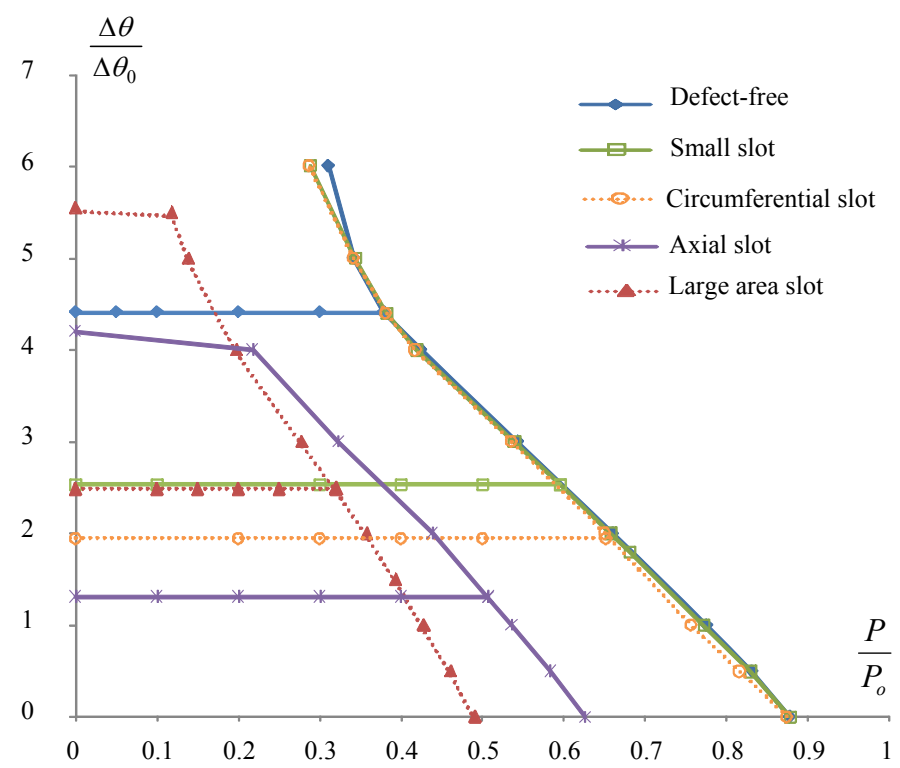

Fig. 6 Shakedown and ratchet limit interaction curve for defective pipeline with shallow type slot 

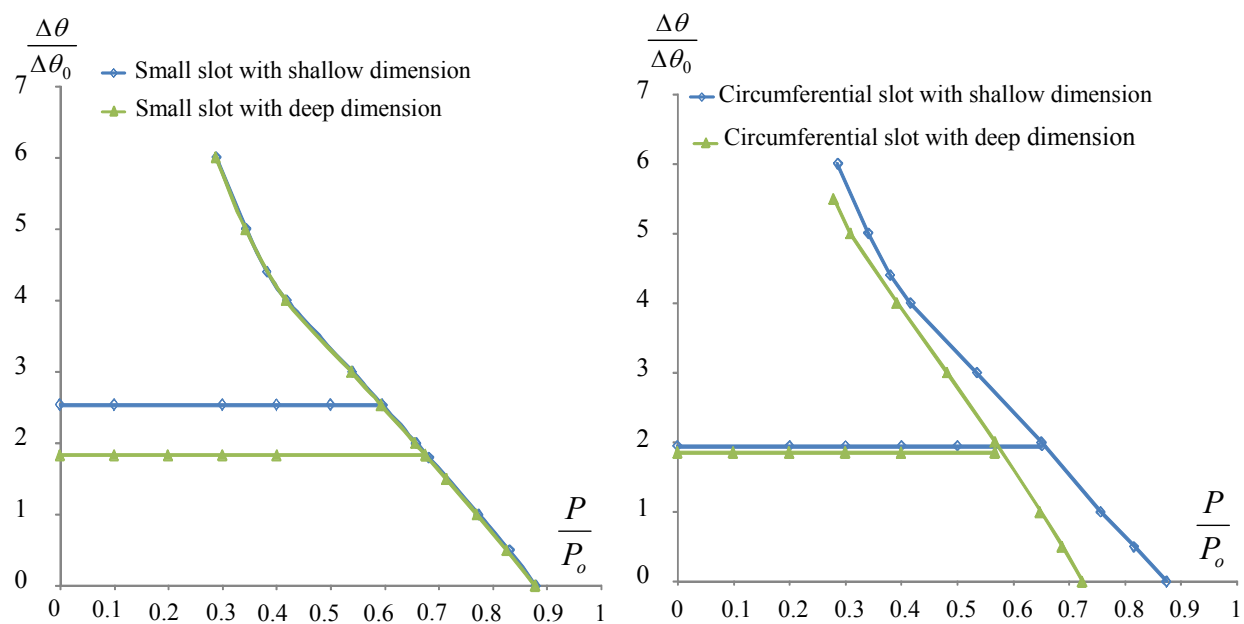

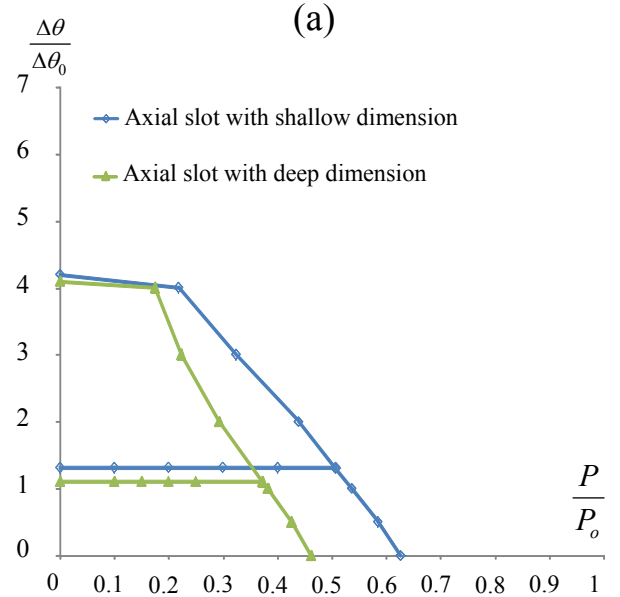

(c)

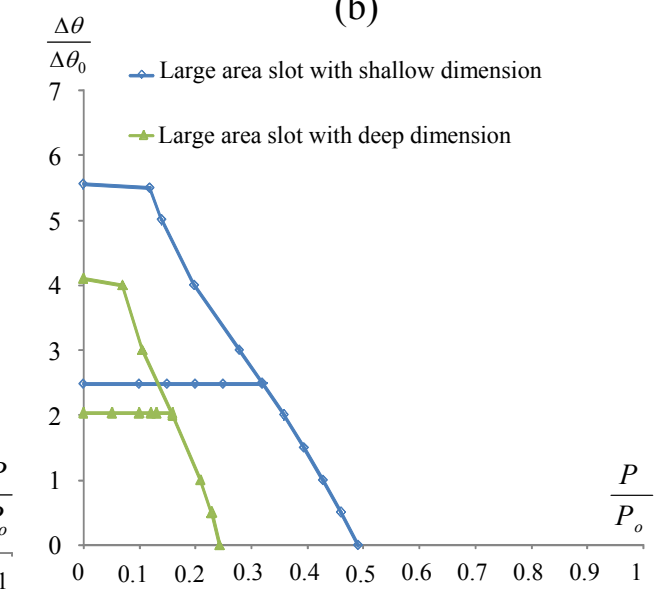

(d)

Fig. 7 Shakedown and ratchet limit interaction curve of part-through slot with different dimensions:

(a)small slot; (b) circumferential slot; (c) axial slot and (d) large area slot 


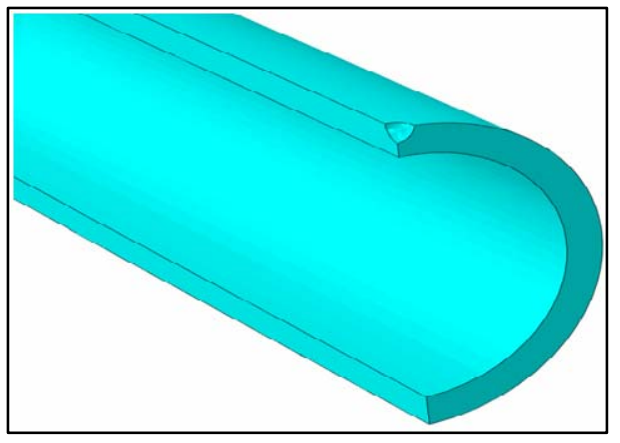

(a) Small slot

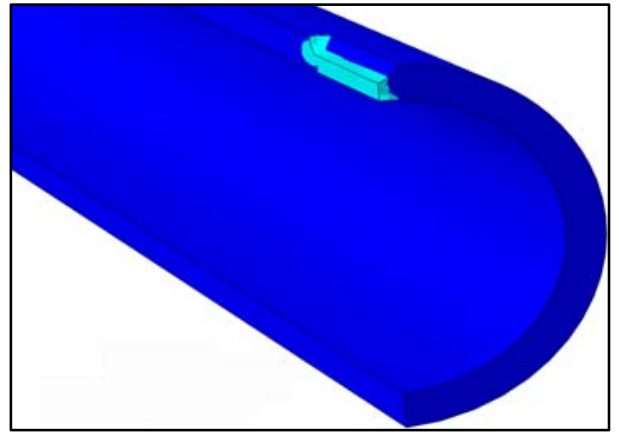

(c) Axial slot

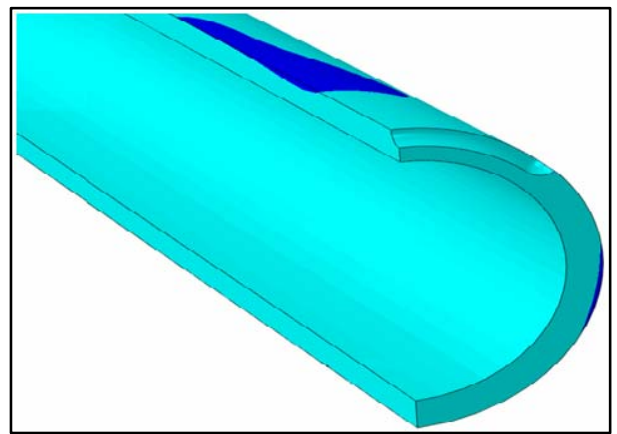

(b) Circumferential slot

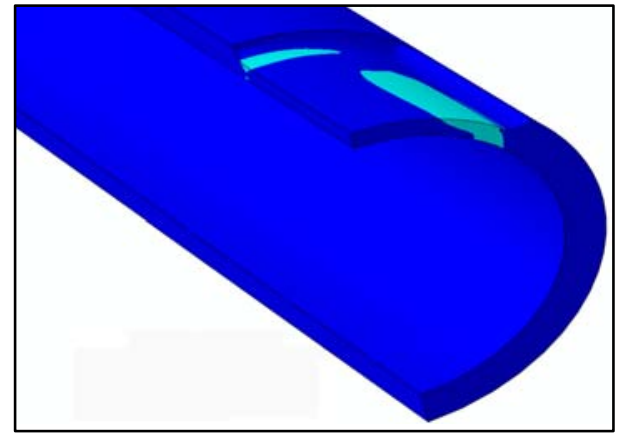

(d) Large area slot

Fig. 8 Failure pattern at the limit state for defective pipeline: (a) small slot; (b) circumferential slot; (c)axial slot and (d)large area slot 


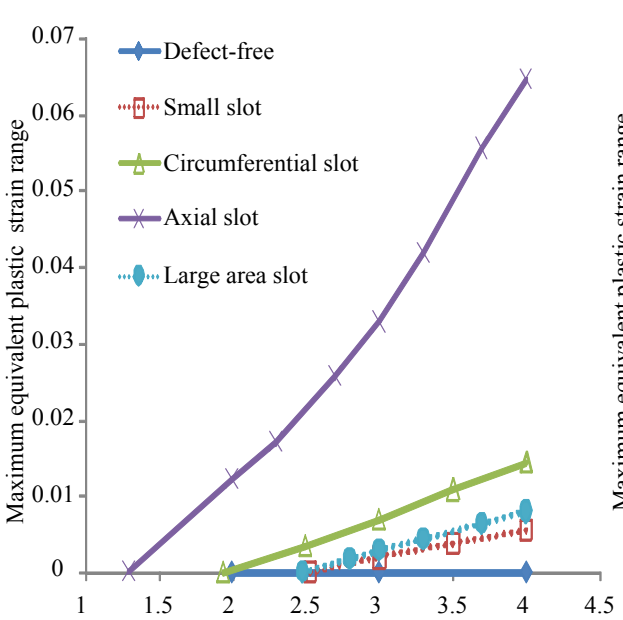

(a)

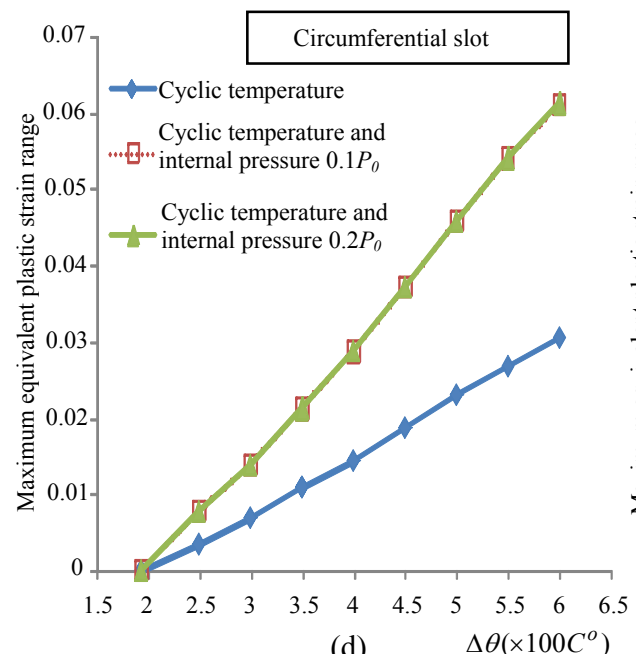

(d)

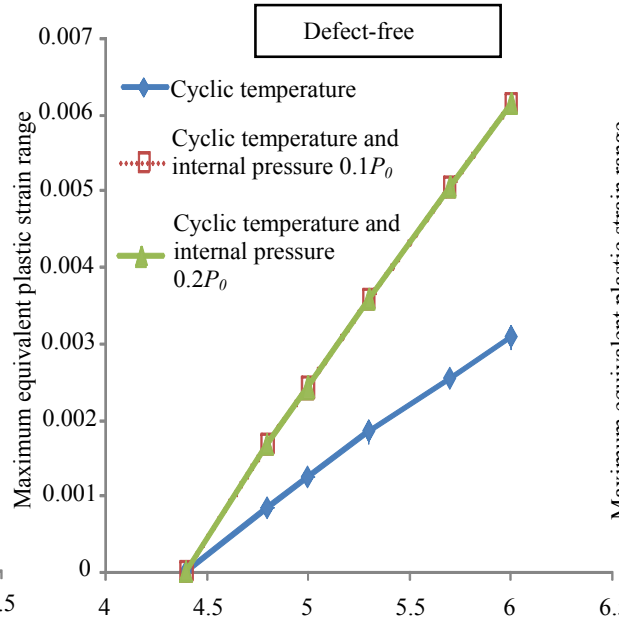

(b) $\quad \Delta \theta\left(\times 100 C^{o}\right)$

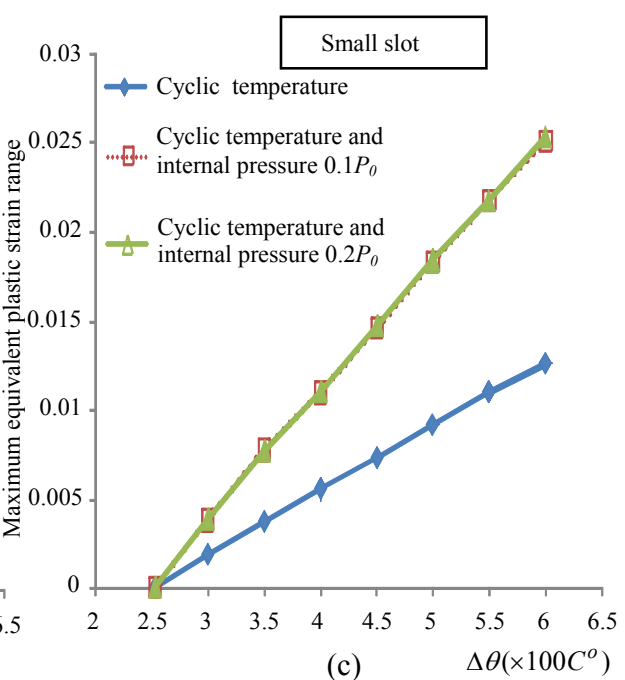

(c)

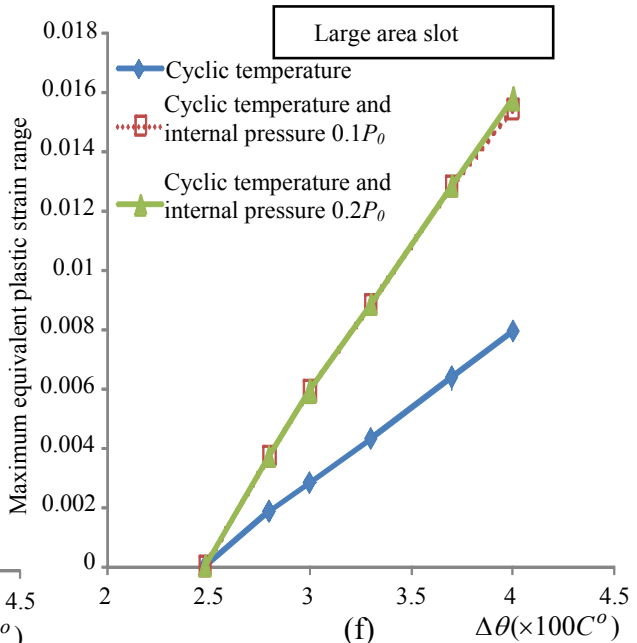

Fig. 9 Maximum equivalent plastic strain range against temperature range for (a) Cyclic thermal load only ( all defect pipeline); (b)Cyclic thermal and mechanical load (defect-free); (c) Cyclic thermal and mechanical load (small slot); (d) Cyclic thermal and mechanical load (circumferential slot); (e) Cyclic thermal and mechanical load (axial slot); (f) Cyclic thermal and mechanical load (large area slot) 


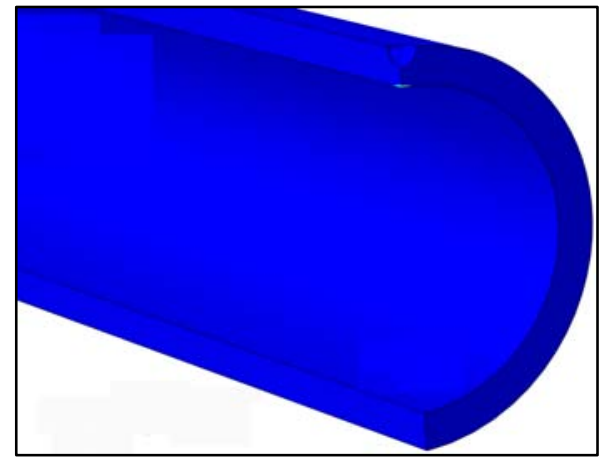

(a) Small slot

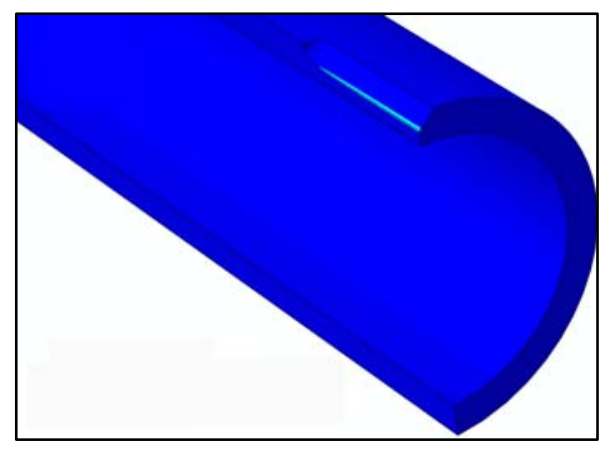

(c) Axial slot

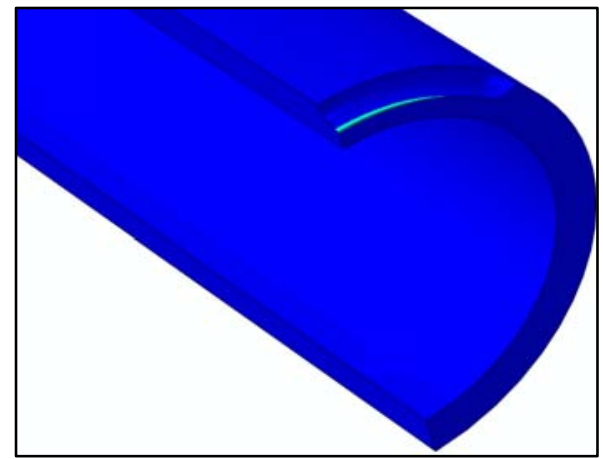

(b) Circumferential slot

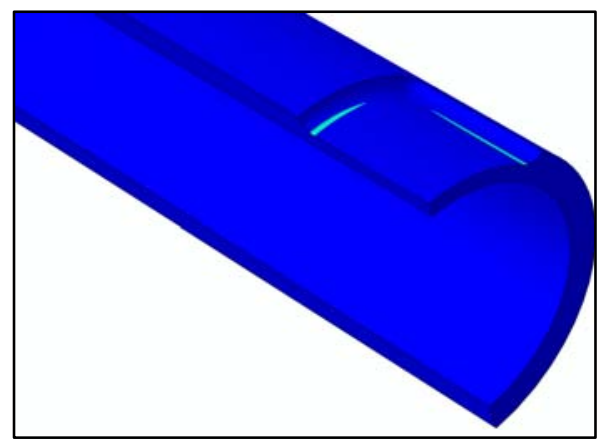

(d) Large area slot

Fig. 10 The location of the initiation of a fatigue crack under cyclic thermal load and constant internal pressure (a)small slot; (b) circumferential slot; (c) axial slot and (d) large area slot 